\title{
EXCESS NOISE FACTOR IN AVALANCHE PHOTODIODES WITH DEAD SPACE EFFECT
}

\begin{abstract}
This project aims to produce a graphical user interface (GUI) for MATLAB programs written by J.S.Marsland as part of his research into the excess noise factor in avalanche photodiodes (APDs). The GUI will be produced using the GUIDE package supplied with the MATLAB software combined with the MATLAB programs. The GUI will then be used to compare this research work with the research work of others e.g. the Monte Carlo calculations made by the research group at the French Aerospace Laboratory (ONERA). Comparison with other research work will require the digitization of some graphs published in academic journals.
\end{abstract}

\section{INTRODUCTION}

Avalanche photodiodes (APDs) amplify photo

generated currents by the process of impact ionization; however this is a random process which results in additional noise quantified by the excess

noise factor. APDs with low excess noise factor and high multiplication (amplification) have many uses in low level light detection such as medical imaging, astronomy, military, etc. Understanding the physics of APDs is very important for the future development of better light detectors.

Avalanche multiplication occurs when energetic carriers create additional carriers by impact ionization. Typically an electron produces different multiplication with impact ionization and is a random process.

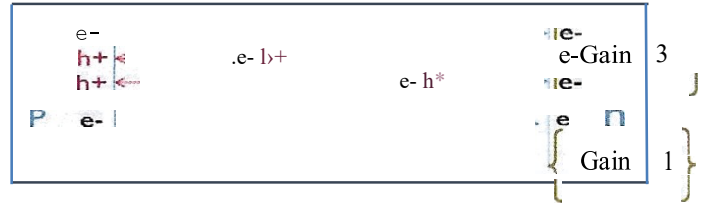

Figure 1. Avalanche multiplication

\section{McINTYRE'S EQUATION}

An ionization coefficient ratio $\mathrm{k}$ is defined such that $\beta$ divided by $\alpha$ and an equation for the excess noise factor is given that relates average multiplication $\mathrm{M}$ with $\mathrm{k}$.

$F(M)=k M+(1-k)\left\{2-\frac{1}{M}\right\} ; k=\frac{\alpha}{\beta}$ (1)

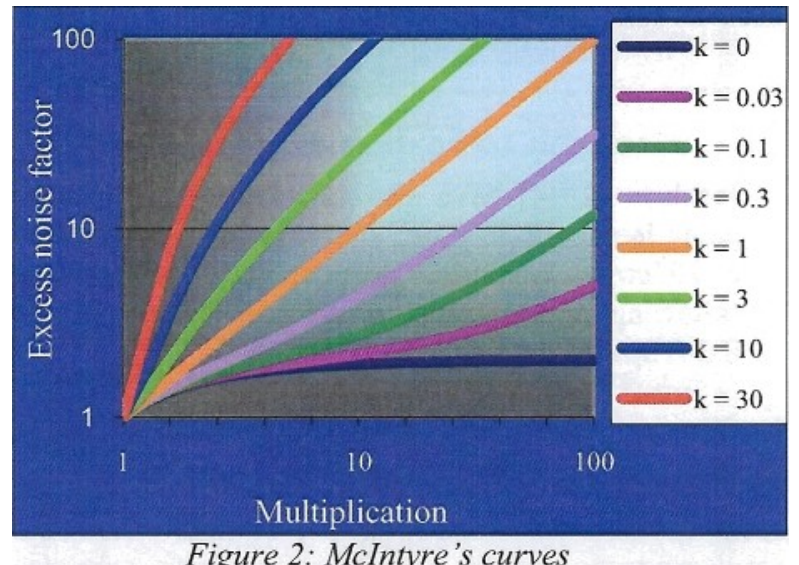

McIntyre [1] made the assumption that the ionization coefficient only depended on the local electric field and did not consider the 'dead space effect'.

\section{NONLOCAL \\ COEFFICIENTS}

IONIZATION

Dead space is nonlocal effect. A nonlocal ionization coefficient $\mathrm{n}(\mathrm{z})$ is defined by Marsland [2] such that $\alpha(z) d z$ is the probability that a carrier startin8 with no kinetic energy at $z=0$ will impact ionize in the interval $(z, z+d z)$.

The ionization path length PDF, $h_{1}(z)$ can be defined such that $h_{1}(z) d z$ is the probability that a carrier will impact ionize for the first time in the given interval.

The probability that a carrier will travel to $\mathrm{z}$ without ionizing is called the survival probability $P_{S}(z)$.

The survival probability can be related to the ionization path length PDF:

$$
P_{S}(z)=1-\int_{0}^{z} h_{1}(x) d x=\int_{z}^{\infty} h_{1}(x) d x
$$

The nonlocal ionization coefficient $n(z)$ is related to the PDF $h_{1}(z)$ :

$$
\alpha(z)=h_{1}(z)+\int_{0}^{z} \alpha(x) h_{1}(z-x) d x
$$

\section{A MODEL FOR IONIZATION PDF}

This behaviour can be described by the following expression where 1 is the length of dead space region and $\mathrm{a}$ and $\mathrm{b}$ are the constants governing the slope of the rise and fall of $h(z)$ :
$h(z)=\frac{a b}{b-a}\{\exp (-a(z-l))-\exp (-b(z-$
l)) $\} U(z-l)$ 
The above equation has been fitted to $h(z)$ calculated using Monte Carlo techniques [4] for electrons in

$G a A s$ at a field of $3 \times 10^{7} \mathrm{vm}^{-1}$ using the following parameters; $a=10 \mu m^{-1}, b=1 \mu m^{-1}$ and $l=0.15 \mu m^{-1}$

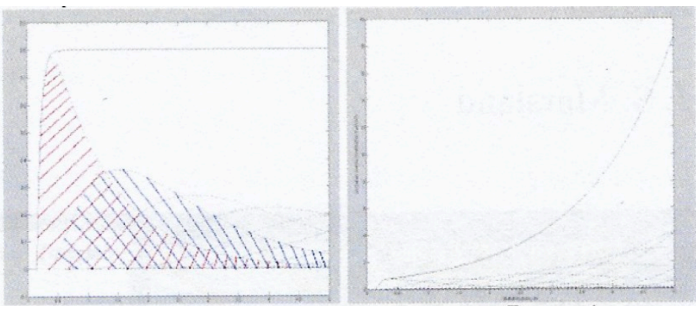

Figure 3: GaAs at $3 \times 10^{7} \mathrm{vm}^{-1}$

Jacob et al. [4] have also calculated the ionization path length PDF for electrons at higher field of $10^{8} \mathrm{vm}^{-1}$. Again this result can be fitted using the

following parameters; $a=b=300 \mu m^{-1}$ and $l=$ $0.0415 \mu \mathrm{m}^{-1}$ :

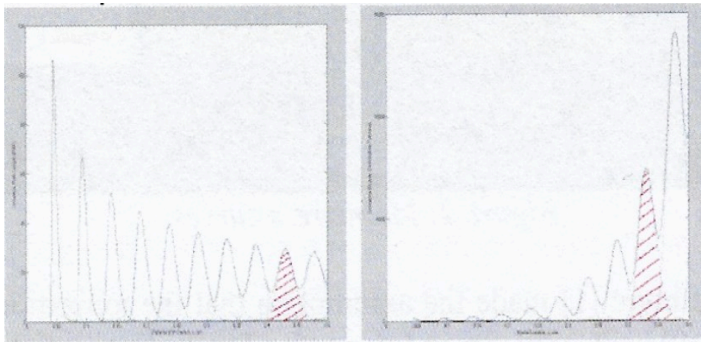

Figure 4: GaAs at $10^{8} \mathrm{vm}^{-1}$

\section{MATLAB GUI}

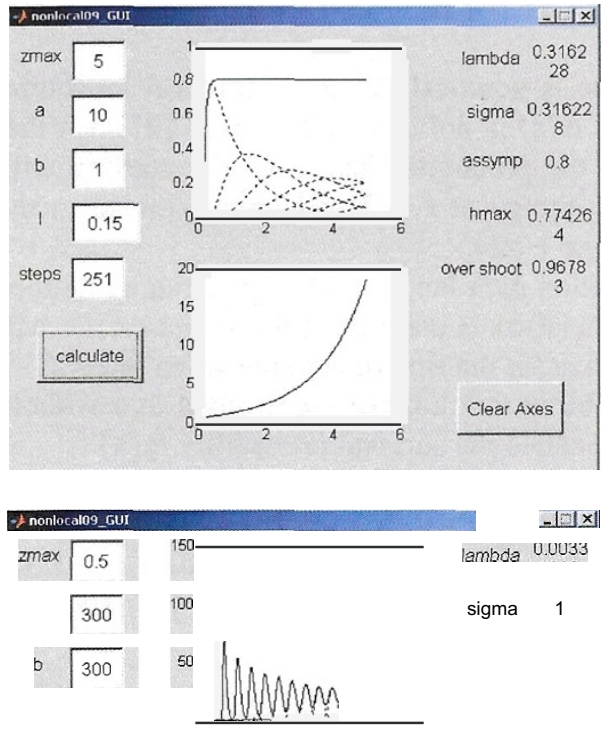

\section{DIGITIZATION}

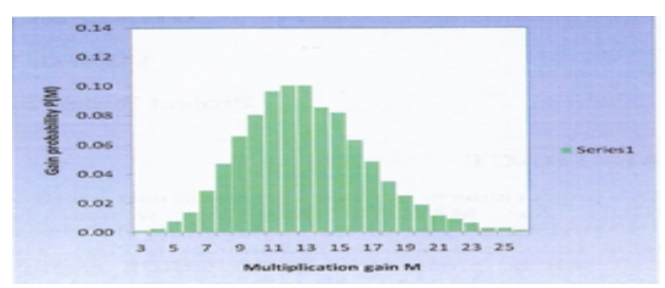

Figure 5: Digitized figure from Derelle et al. [3]

Probability, $\sum P(M)=1$

Mean multiplication, $\sum M P(M)=\langle M\rangle=12.02$

Mean square, $\left\langle M^{2}\right\rangle=\sum M^{2} P(M)=$

168

Excess noise factor, $\frac{\left\langle M^{2}\right\rangle}{\langle M\rangle^{2}}=1.162$

Comparison of MJ03 with Derelle et al. [31

\begin{tabular}{|c|c|}
\hline MJ03 & Monte Carlo \\
\hline$(\mathrm{M})=12.02$ & $(\mathrm{M})=13.07$ \\
\hline $\mathrm{F}=1.162$ & $\mathrm{~F}=1.052$ \\
\hline
\end{tabular}

\section{CONCLUSION}

Ideally we would want the APD to have maximum multiplication and less noise. This scenario is shown using nonlocal ionization coefficients which gives stable multiplication and reduced noise. When dead space is included noise is considerably reduced and is lesser than the noise predicted by McIntyre's curves. A model for ionization PDF is developed and is reprcsented using a GUI for MATLAB program written by J.S.Marsland. 


\section{REFERENCES}

[1] R.J. McIntyre, IEEE Trans. Electron Devices, ED13, 164(1966). Doi: 10.1109/T-ED. 1966. 1565 I

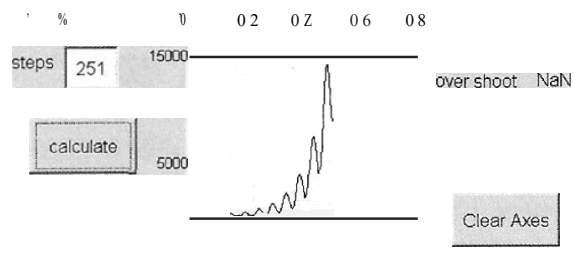

The output graphs of the GUI obtained are as expected although the dotted lines of the axes component 2 is not displayed.
[2] J.S. Marsland, Electron. Lett. 38, 55 (2002). Doi: 10.1049/el:20020025

[3] Derelle S, Bernhardt S, Haidar R, Primot I, Deschamps I and Rothman I, IEEE Trans. Electron Devices, Vol. 56,.No. 4, p569. Doi:

10.1109/TED.2009.2012526

[4] B. Jacob, S.A. Plimmer, P.N. Robson, G.J. Rees, IEE Proc. Optoelectron. 148, 81 (2001). Doi: 10.1049/ip-opt:20010089 